\title{
Efficacy of Gene Modification in Placenta-Derived Mesenchymal Stem Cells Based on Nonviral Electroporation
}

\author{
Jae Yeon Kim ${ }^{1, *}$, Jong Ho Choi ${ }^{2, *}$, Se Ho Kim ${ }^{1}$, Hyeri Park ${ }^{1}$, Dongsook Lee ${ }^{3}$, Gi Jin Kim ${ }^{1}$ \\ ${ }^{1}$ Department of Biomedical Science, CHA University, Seongnam, Korea \\ ${ }^{2}$ Department of Oral Pathology, College of Dentistry, Gangneung-Wonju National University, Gangneung, Korea \\ ${ }^{3}$ Hamchoon Women's clinic, Research Center of Fertility \& Genetics, Seoul, Korea
}

Mesenchymal stem cell (MSC)-based therapy using gene delivery systems has been suggested for degenerative diseases. Although MSC-based clinical applications are effective and safe, the mode of action remains unclear. Researchers have commonly applied viral-based gene modification because this system has efficient vehicles. While viral transfection carries many risks, such as oncogenes and chromosomal integration, nonviral gene delivery techniques are less expensive, easier to handle, and safe, although they are less efficient. The electroporation method, which uses Nucleofection technology, provides critical opportunities for hard-to-transfect primary cell lines, including MSCs. Therefore, to improve the therapeutic efficacy using genetically modified MSCs, researchers must determine the optimal conditions for the introduction of the Nucleofection technique in MSCs. Here, we suggest optimal methods for gene modification in PD-MSCs using an electroporation gene delivery system for clinical application.

Keywords: Electroporation, Gene modification, Placenta-derived mesenchymal stem cells, Nonviral gene delivery system

\section{Introduction}

Many studies have emphasized the clinical importance of mesenchymal stem cells (MSCs) in degenerative and regenerative medicine. Recently, extensive clinical trials of MSCs have been conducted. However, their efficacy depreciates because of poor survival and engraftment in injured organs. In particular, the therapeutic mechanism or

Received: July 21, 2020, Revised: October 28, 2020,

Accepted: October 28, 2020, Published online: December 31, 2020 Correspondence to Gi Jin Kim

Department of Biomedical Science, CHA University, 335 Pangyo-ro, Bundang-gu, Seongnam 13488, Korea

Tel: +82-31-881-7234, Fax: +82-31-881-7249

E-mail: gjkim@cha.ac.kr

${ }^{*}$ These authors contributed equally to this work.

(a) This is an open-access article distributed under the terms of the Creative Commons Attribution Non-Commercial License (http://creativecommons.org/ licenses/by-nc/4.0/), which permits unrestricted non-commercial use, distribution, and reproduction in any medium, provided the original work is properly cited.

Copyright (c) 2021 by the Korean Society for Stem Cell Research "mode of action" is lacking. MSC-based therapy using a gene delivery system shows enhanced efficacy due to improvement in cell survival and secretion of numerous growth factors (1). In general, viral transfection has advantages such as high efficiency in genetic transfer and stable gene expression. Previously, an optimal protocol was developed using lentiviral transfection in bone marrow-derived MSCs (BM-MSCs), resulting in higher efficiency and lower cell toxicity $(2,3)$. However, viral vectors have several limitations, including their risk of immunogenicity and chromosomal integration in clinical applications (4). The current strategy, utilizing Nucleofection ${ }^{\mathrm{TM}}$ for the nonviral gene delivery, is not only acceptable with regard to safety issues but also is easy to handle, indicating possible applications in patient. In particular, this method has been successfully applied to difficult-to-transfect primary cells, including MSCs. Nucleofection ${ }^{\mathrm{TM}}$ platforms could potentially affect the innate abilities of MSCs (5). In our previous reports, naïve placenta-derived MSCs (PD-MSCs) were transfected using Nucleofection ${ }^{\mathrm{TM}}$ and subjected to genetic analyses (6). However, the Nucleofection ${ }^{\mathrm{TM}}$ non- 
viral gene delivery in MSCs is still being developed, requiring their optimization to safely establish a cell line, maintain the characteristics of naïve MSCs, and promote therapeutic efficacy. In this report, we will highlight the optimal protocol for the generation of functionally enhanced MSCs using a nonviral gene delivery system.

\section{Materials and Methods}

\section{Primary cells}

Naïve PD-MSCs were isolated as previously described using placentas for all participating women at term $(\geq 37$ gestational weeks) (7). The protocol was approved by the Institutional Review Board (IRB) of CHA General Hospital, Seoul, Republic of Korea (IRB 07-18).

- Note: Naïve PD-MSCs were maintained in alpha-modified minimum essential medium ( $\alpha$-MEM; HyClone, USA) supplemented with $10 \%$ fetal bovine serum (FBS; Gibco, USA), 1\% penicillin/streptomycin (P/S; Gibco), $25 \mathrm{ng} / \mathrm{ml}$ human fibroblast growth factor-4 (hFGF4; PeproTech, USA), and $1 \mu \mathrm{g} / \mathrm{ml}$ heparin (SigmaAldrich, USA).

\section{Reagents}

- $\alpha$-MEM (cat. no. SH30265.01)

- FBS (cat. no. GIB-16000-044)

- hFGF4 (cat. no. 100-31-100)

- Heparin (cat. no. H3149)

- P/S (cat. no. GIB-15140-122)

- Dulbecco's phosphate buffered saline (DPBS; Wellgene, cat. no. LB001-02, Korea)

- Phosphatase of regenerating liver-1 (PRL-1) plasmid (Origene, Inc., cat. no. RG200435, USA)

- pCMV-AC-GFP (Origene, Inc., cat. no. PS100010)

- TrypLE ${ }^{\mathrm{TM}}$ Express (+) Phenol Red (Gibco, cat. no. GIB-12605-010)

- Trypan blue, 0.4\% (Gibco, cat. no. GIB-15250-061)

- P1 Primary Cell 4D Nucleofector ${ }^{\mathrm{TM}}$ X Kit L (Lonza, cat. no. V4XP-1024, Switzerland)

- Note: Including P1 Primary Cell Nucleofector ${ }^{\mathrm{TM}}$ Solution, Supplement 1, $50 \mu \mathrm{g}$ pmaxGFP ${ }^{\mathrm{TM}}$ Vector $(1 \mu \mathrm{g} / \mu \mathrm{l}$ in $10 \mathrm{mM}$ Tris $\mathrm{pH} 8.0)$

- Neomycin (Sigma-Aldrich, cat. no. N6386)

\section{Equipment}

- 4D-Nucleofector ${ }^{\mathrm{TM}}$ Core Unit (Lonza, cat. no. AAF1002B)

- 4D-Nucleofector ${ }^{\mathrm{TM}} \mathrm{X}$ Unit (Lonza, cat. no. AAF-1002X)

- Nucleocuvette ${ }^{\mathrm{TM}}$ Vessels (Lonza, cat. no. AXP-1003)

- T75 cell culture flask (SPL, cat. no. 70075, Korea)
- $100 \mathrm{~mm}$ cell culture dish (Nunc, cat. no. 150466, Denmark)

- Hemocytometer (Marienfeld, cat. no. 0610039094, Germany)

- Conical tube, $15 \mathrm{ml}$ (SPL, cat. no. 50015, Korea)

- Centrifuge (Labogene, cat. no. 1730R, Korea)

- $\mathrm{CO}_{2}$ incubator (Thermo Scientific, Hera Cell, cat. no. $\mathrm{H} 150-\mathrm{CO}_{2}$, USA)

Pre Nucleofection ${ }^{\mathrm{TM}}$ : 1.1 Seed naïve PD-MSCs $(2.5 \times$ $10^{5}$ cells) in a T75 cell culture flask containing $15 \mathrm{ml}$ of cell culture medium and incubate overnight at $37^{\circ} \mathrm{C}$ in a $5 \% \mathrm{CO}_{2}$ incubator.

1.2 Naïve PD-MSCs (passage $<9$ ) should reach approximately $80 \%$ confluence.

- Note: Transfection using naïve PD-MSCs may be donor-dependent. Thus, it is recommended to use passages earlier than 9.

1.3 Remove the medium from the cultured naïve PDMSCs and wash the cells with DPBS 2 times.

1.4 Add $2 \mathrm{ml}$ of TrypLE ${ }^{\mathrm{TM}}$ Express (+) Phenol Red and incubate the cells for $2 \mathrm{~min}$ at $37^{\circ} \mathrm{C}$ in a $5 \% \quad \mathrm{CO}_{2}$ incubator.

1.5 Harvest cells with prewarmed complete medium and obtain an aliquot of cells.

1.6 Count the Trypan blue-stained cells using hemocytometer and divide the cell suspension $\left(5 \times 10^{5}\right.$ cells/tube $)$ in each $15 \mathrm{ml}$ conical tube.

1.7 Centrifuge at $200 \mathrm{x} \mathrm{g}$ for $10 \mathrm{~min}$ at room temperature.

- Note: To avoid the cell damage, speed should not exceed $200 \mathrm{x}$ g.

Nucleofection $^{\mathrm{TM}}: 2.1$ Ensure that the entire supplement solution is added to the Nucleofection ${ }^{\mathrm{TM}}$ solution.

- Note: The ratio of the Nucleofection ${ }^{\mathrm{TM}}$ solution to the supplement solution is $4.5: 1$. The mixture is stable for 3 months at $4^{\circ} \mathrm{C}$. For a single reaction, mix $82 \mu 1$ of Nucleofection $^{\mathrm{TM}}$ solution and $18 \mu 1$ of supplement solution.

2.2 Operate the 4D Nucleofector ${ }^{\mathrm{TM}}$ System and set the appropriate Nucleofector ${ }^{\mathrm{TM}}$ program.

- Note: Turn on the 4D Nucleofector ${ }^{\mathrm{TM}}$ System $\rightarrow$ choose a X unit device $\rightarrow$ choose a cuvette vessel $\rightarrow$ choose the number of cuvettes $\rightarrow$ Select a cell type program "MSC, human" $\rightarrow$ pulse code was set up as FF-104 $\rightarrow$ press $\mathrm{OK}$

- Note: Pulse code as FF-104 for high efficiency.

2.3 Prepare complete medium in $100 \mathrm{~mm}$ cell culture dish and preincubate/equilibrate each plate without cells at $37^{\circ} \mathrm{C}$ in a $5 \% \mathrm{CO}_{2}$ incubator.

- Note: For the stabilization of transfected PD-MSCs, its step is important to improve transfection efficiency and viability. 
2.4 After centrifugation at $200 \mathrm{x}$ g for $10 \mathrm{~min}$ at room temperature, carefully resuspend the cell pellet in $100 \mu 1$ of Nucleofection ${ }^{\mathrm{TM}}$ solution.

2.5 Add an appropriate volume of plasmid in each tube and mix well.

- Note: pmaxGFP ${ }^{\mathrm{TM}}$ Vector $\left(2 \mu \mathrm{g} / 5 \times 10^{5}\right.$ cells $/ 100 \mu \mathrm{l}$ Nucleofection $^{\text {TM }}$ solution $100 \mu \mathrm{l} /$ cuvette).

- Note: PRL-1/pCMV-AC-GFP $\left(5 \mu \mathrm{g} / 5 \times 10^{5}\right.$ cells/100 $\mu \mathrm{l}$ Nucleofection $^{\mathrm{TM}}$ solution $100 \mu \mathrm{l}$ /cuvette).

- Note: Make sure that matermixtures must mix well while pipetting.

- Note: Volume of mastermixtures should not exceed maximum $10 \%$ of the total reaction volume $(10 \mu 1$ for $100 \mu 1$ reaction). It is recommended that the concentration of plasmid is suitable for $1 \mu \mathrm{g} / \mu \mathrm{l}$.

2.6 Transfer mastermixtures into the Nucleocuvette ${ }^{\mathrm{TM}}$ Vessels and place Nucleocuvette ${ }^{\mathrm{TM}}$ Vessels in the retainer of the 4D Nucleofector ${ }^{\mathrm{TM}} \mathrm{X}$ unit device.

- Note: In order to prevent an electrical signal error, avoid air bubbles while transferring mastermixtures.

2.7 Start Nucleofection process according to the presetting value (refer to 2.2 )

2.8 After running, remove Nucleocuvette ${ }^{\mathrm{TM}}$ Vessels in the retainer of the device and incubate Nucleocuvette ${ }^{\mathrm{TM}}$ for $10 \mathrm{~min}$ at room temperature.

2.9 Add $500 \mu 1$ of prewarmed PD-MSCs medium and gently resuspend the transfected cells.

2.10 Plate the transfected cells in preincubated cell culture plates.

Post Nucleofection ${ }^{\mathrm{TM}}$ : 3.1 Incubate the transfected cells at $37^{\circ} \mathrm{C}$ in a $5 \% \mathrm{CO}_{2}$ incubator for $24 \mathrm{~h}$.

- Note: Gene expression is often detectable after $4 \sim 8$ h. However, gene or GFP expression in PD-MSCs recommended $24 \mathrm{~h}$ post Nucleofection ${ }^{\mathrm{TM}}$.

3.2 After $24 \mathrm{~h}$, remove medum and change selection medium including $1.5 \mathrm{mg} / \mathrm{ml}$ neomycin for 7 days.

3.3 Confirm the gene or GFP expression.

Table. 1 Primer sequences using RT- and qRT-PCR

\begin{tabular}{|c|c|c|c|}
\hline Genes & & Primer sequences & $\mathrm{Tm}$ \\
\hline \multirow[t]{2}{*}{ Oct4 } & Forward & 5'-AGTGAGAGGCAACCTGGAGA-3' & 52 \\
\hline & Reverse & 5'-GTGAAGTGAGGGCTCCCATA-3' & \\
\hline \multirow[t]{2}{*}{ Nanog } & Forward & 5'-ТTCTTGACTGGGACCTTGTC-3' & 52 \\
\hline & Reverse & 5'-GCTTGCCTTGCTTTTGAAGCA-3' & \\
\hline \multirow[t]{2}{*}{ Sox 2} & Forward & 5'-GGGCAGCGTGTACTTATCCT-3' & 52 \\
\hline & Reverse & 5'-AGAACCCCAAGATGCACAAC-3' & \\
\hline \multirow[t]{2}{*}{ TERT } & Forward & 5'-GAGCTGACGTGGAAGATGAG-3' & 55 \\
\hline & Reverse & 5'-CTTCAAGTGCTGTCTGATTCCAATG-3' & \\
\hline \multirow[t]{2}{*}{ HLA-G } & Forward & 5'-GCGGCTACTACAACCAGAGC-3' & 58 \\
\hline & Reverse & 5'-GCACATGGCACGTGTATCTC-3' & \\
\hline \multirow[t]{2}{*}{$\beta$-actin } & Forward & 5'-TCСТTCTGCATCСТGTCAGCA-3' & 58 \\
\hline & Reverse & 5'-CAGGAGATGGCCACTGCCGCA-3' & \\
\hline \multirow[t]{2}{*}{ Human Alu } & Forward & 5'-GGAGGCTGAGGCAGGAGAA-3' & 55 \\
\hline & Reverse & 5'-CGGAGTCTCGCTCTGTCGCССA-3' & \\
\hline \multirow[t]{2}{*}{ BGLAP } & Forward & 5'-САСТССТСGСССТАТTGGC & 58 \\
\hline & Reverse & 5'-СССТССТGСТTGGACACAAAG-3' & \\
\hline \multirow[t]{2}{*}{ COL1A1 } & Forward & 5'- AGACATCCCACCAATCACCT-3' & 60 \\
\hline & Reverse & 5'-CGTCATCGCACAACACCT-3' & \\
\hline \multirow[t]{2}{*}{ Adipsin } & Forward & 5'-GGTCACCCAAGCAACAAAGT-3' & 60 \\
\hline & Reverse & 5'-ССТCСТGCGTTCAAGTCATC-3' & \\
\hline \multirow[t]{2}{*}{ PPARG } & Forward & 5'-TTGACCCAGAAAGCGATTCC-3' & 60 \\
\hline & Reverse & 5'-AAAGTTGGTGGGCCAGAATG-3' & \\
\hline \multirow[t]{2}{*}{ Albumin } & Forward & 5'-TGAGTTTGCAGAAGTTTCCA-3' & 60 \\
\hline & Reverse & 5'-CCTTTGCCTCAGCATAGTTT-3' & \\
\hline \multirow[t]{2}{*}{ TAT } & Forward & 5'-AACGATGTGGAGTTCACGG-3' & 58 \\
\hline & Reverse & 5'-GACACATCCTCAGGAGAATGG-3' & \\
\hline \multirow[t]{2}{*}{ PRL-1 } & Forward & 5'-TACTGCTCCACCAAGAAGCC-3' & 60 \\
\hline & Reverse & 5'-AGGTTTACCCCATCCAGGTC-3' & \\
\hline \multirow[t]{2}{*}{ GAPDH } & Forward & 5'-GCACCGTCAAGGCTGAGAAC-3' & 60 \\
\hline & Reverse & 5'-GTGGTGAAGACGCCAGTGGA-3' & \\
\hline
\end{tabular}




\section{Characterizations}

Cell viability and transfection efficiency using quantitative real-time polymerase chain reaction (qRT-PCR) and western blotting: PRL-1-overexpressing PD-MSCs (PD-MSCs ${ }^{\text {PRL-1 }}$, PRL-1 + ) were counted 24 h post transfection to examine the transfected cell viability. For analysis of the efficiency of PD-MSCs ${ }^{\text {PRL-1 }}$ post-transfection, total RNA and protein lysate were extracted from the cell pellet. qRT-PCR analysis was performed with primers (Table 1) using the CFX Connect ${ }^{\mathrm{TM}}$ Real-Time System (Bio-Rad, USA). Western blotting was performed with primary monoclonal anti-turboGFP (1:500, Origene, Inc.) and horseradish peroxidase (HRP)-conjugated anti-goat IgG (Santa Cruz Biotechnology, USA).

Expression of stemness markers by reverse transcription (RT)-PCR: For analysis of stemness markers in PD-MSCs ${ }^{\text {PRL-1 }}$, PCR amplification was conducted with Taq DNA polymerase (Solgent, Korea) according to the manufacturer's instructions. Each primer is provided in Table 1.

Immunophenotypes by flow cytometry: For analysis of the immunophenotyping of cell surface antigens, PD$\mathrm{MSCs}^{\text {PRL-1 }}$ cells were stained with phycoerythrin (PE)conjugated CD13, CD90, and CD105 (BD Bioscience, USA) and fluorescein isothiocyanate (FITC)-conjugated HLA-ABC, HLA-DR (BD Bioscience), and HLA-G (Abcam, UK) using a CytoFLEX flow cytometer (Beckman Coulter, USA). All data sets were collected from 10,000 cells.

Differentiation potential: Naïve PD-MSCs were previously confirmed for their multilineage differentiation ability (8). PD-MSCs ${ }^{\text {PRL-1 }}$ were plated $\left(5 \times 10^{3}\right.$ cells $\left./ \mathrm{cm}^{2}\right)$ in differentiation induction medium using a StemPro Adipogenesis and Osteogenesis differentiation kit (Gibco) as well as hepatogenic differentiation as previously described (9). After each differentiation, cells were stained with oil red O (Sigma-Aldrich) for adipogenesis, von Kossa (Sigma-Aldrich) for osteogenesis, and $1 \mathrm{mg} / \mathrm{ml}$ indocyanine green (ICG) (Dong in Dang Pharm., Korea) for uptake.

Karyotyping: For analysis of karyotypes of naive and PD-MSC ${ }^{\text {PRL-1 }}$, G-banding techniques were performed, and the karyotype was elucidated by the International System for Human Cytogenomic Nomenclature (ISCN 2016) using an Axipskop 2 plus light microscope (Zeiss, Germany).

Teratoma formation: For analysis of teratoma formation by PD-MSCs ${ }^{\text {PRL-1 }}, 5 \times 10^{5}$ cells were transplanted into one testis $(\mathrm{Tx} ; \mathrm{n}=2)$ while another testis was not injected (Con; $n=2$ ) in nine-week-old male nonobese diabetic/severe combined immunodeficiency (NOD/SCID) mice (Laboratory Animal Research Center, Bundang CHA Medical Center, Seongnam, Korea). After 14 weeks, the mice were sacrificed, testis tissues were stained with hematoxylin \& eosin (H\&E), and qRT-PCR analysis was performed with the human Alu sequence (Table 1).

\section{Results}

\section{Optimization of transfection in PD-MSCs}

Naïve PD-MSCs were transfected using the nonviral AMAXA electroporation technique as well as the lentiviral
A

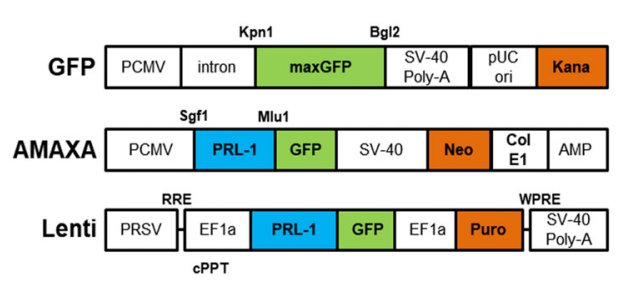

B

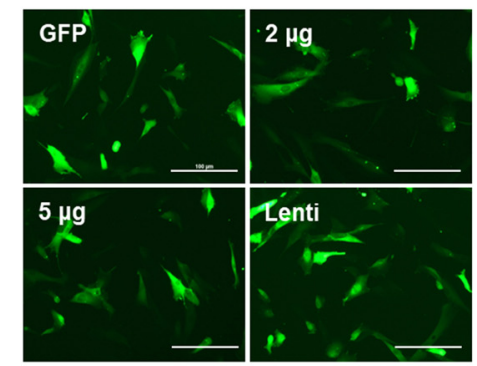

C

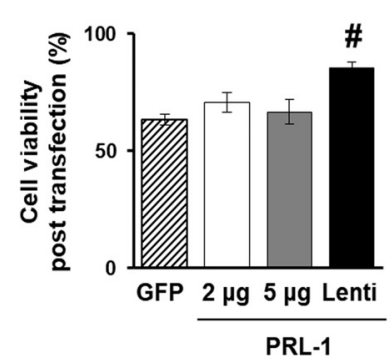

D

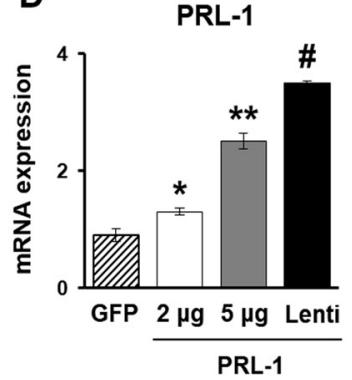

E

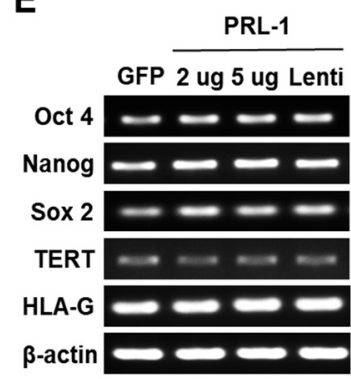

Fig. 1. Optimization of transfection in PD-MSCs. (A) Maps of GFP, lentiviral, and nonviral plasmid vectors. (B) GFP expression using 2 and $5 \mu \mathrm{g}$ of PRL-1 plasmid using nonviral electroporation as well as the lentiviral system. Scale bar $=100 \mu \mathrm{m}$. (C) Cell viability at $24 \mathrm{~h}$ after transfection. (D) mRNA expression of PRL-1 in PD-MSCs ${ }^{\text {PRL-1 }}{ }^{*} \mathrm{p}<0.05$ versus GFP; ${ }^{* *} \mathrm{p}<0.05$ versus $2 \mu \mathrm{g}$; $\# p<0.05$ versus lentiviral system. (E) Stemness markers (e.g., Oct4, Nanog, Sox2), TERT, and HLA-G expression in PD-MSCs ${ }^{\text {PRL-1 }}$ were determined by RT-PCR. Data from each group are expressed as the mean \pm SD. 
system as a positive control group for PRL-1 gene expression (Fig. 1A). To optimize the concentration of PRL-1 plasmid using nonviral electroporation, we used both 2 and $5 \mu \mathrm{g}$, and GFP positivity was indicated (Fig. 1B). Cell viability in the 2 and $5 \mu \mathrm{g}$ groups was not significantly different (Fig. 1C). mRNA expression of PRL-

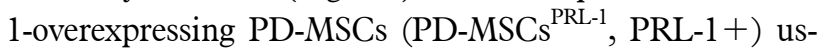
ing both gene delivery systems was increased. In electroporation system, $5 \mu \mathrm{g}$ of PRL-1 resulted in significantly higher effects than $2 \mu \mathrm{g}$ of PRL-1 (Fig. 1D) as well as PRL-1-overexpressing bone marrow-derived MSCs (BM$\mathrm{MSCs}^{\mathrm{PRL}-1}$ ) (Supplementary Fig. S1). Additionally, stemness markers (e.g., Oct4, Nanog, Sox2), telomerase reverse transcriptase (TERT), and immunomodulatory factor HLA-G expression was maintained regardless of the concentration of the PRL-1 plasmid (Fig. 1E). Therefore, these data suggest that $5 \mu \mathrm{g}$ of transfected PD-MSCs ${ }^{\text {PRL-1 }}$ using a nonviral system is as efficient as the lentiviral system.

\section{Characterization of PD-MSCs ${ }^{\mathrm{PRL}-1}$ using a nonviral electroporation system}

In the cultured supernatant, the secreted PRL-1 level was significantly higher than that in naïve cells (Fig. 2A). Additionally, PRL-1 transfection with the GFP fusion protein was confirmed (Fig. 2B). PD-MSCs ${ }^{\text {PRL-1 }}$ were confirmed by surface markers in flow cytometry. Similar to the naïve patterns, the expression of MSC markers (e.g., CD13, CD90, CD105, HLA-ABC, and HLA-G) in PD-MSCs ${ }^{\text {PRL-1 }}$ was positive, whereas HLA-DR was negative (Fig. 2C). To analyze the differentiation abilities of PD-MSCs ${ }^{\text {PRL-1 }}$, we confirmed mesodermal (osteogenic and adipogenic) and endodermal (hepatogenic) lineages using von Kossa, oil red O, and ICG staining, respectively (Fig. $2 \mathrm{D} \sim \mathrm{F}$ ). In addition, the expression of osteogenic (e.g., bone gamma carboxyglutamic acid containing protein; BGLAP and collagen type 1 alpha 1; COL1A1), adipogenic (e.g., adipsin and peroxisome proliferator activated receptor gamma; PPARG), and hepatogenic (e.g., albumin and tyrosine aminotransferase; TAT) markers in differentiated PD-MSCs ${ }^{\text {PRL-1 }}$ were significantly increased compared to those of the undifferentiated PD-MSCs ${ }^{\text {PRL-1 }}$ as shown by qRT-PCR analysis $\left({ }^{*} \mathrm{p}<0.05\right)$. Thus, these findings indicate that PD-MSCs ${ }^{\text {PRL-1 }}$ retain the potential to differentiate into multiple lineages.

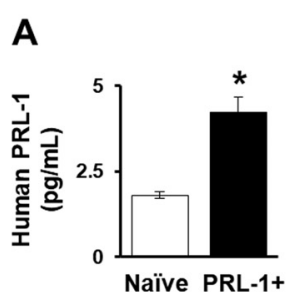

C
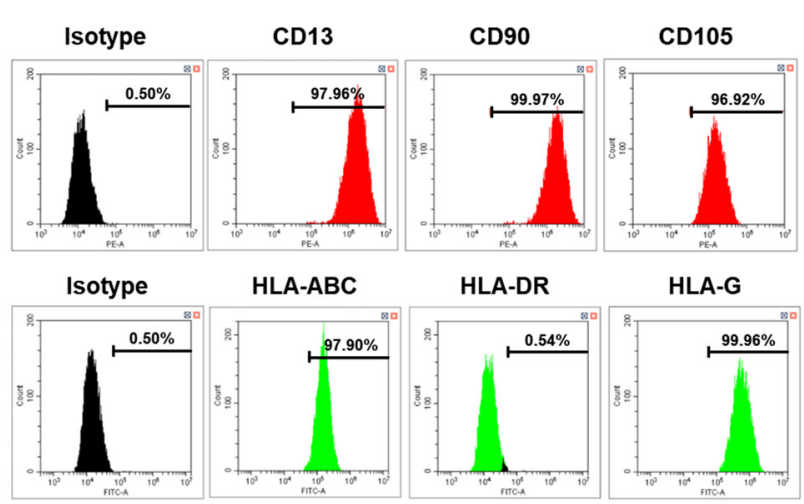

B

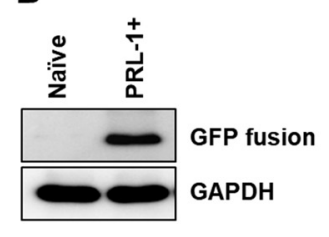

D

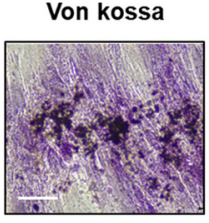

E

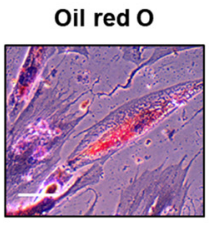

$\mathbf{F}$

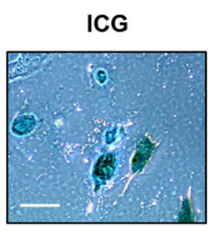

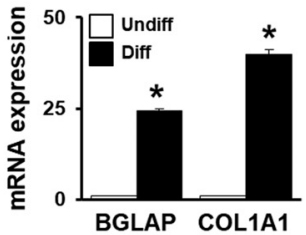
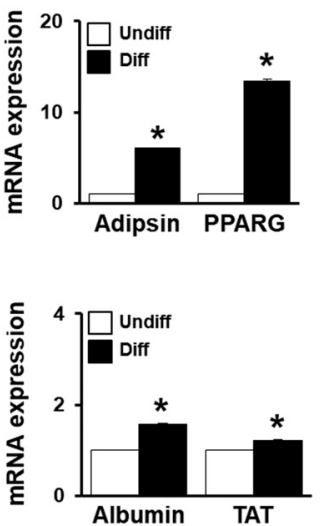

Fig. 2. Characterization of PD-MSCs ${ }^{\text {PRL-1 }}$ using a nonviral electroporation system. (A) Hydrophobic PRL-1 levels in the cell culture supernatant. ${ }^{*} \mathrm{p}<0.05$ versus naïve. (B) GFP fusion protein expression after transfection shown by western blotting. (C) FACS analysis of surface markers

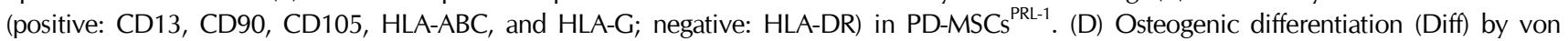
Kossa staining and mRNA of BGLAP and COL1A1. (E) Adipogenic differentiation by oil red O staining and mRNA of adipsin and PPARG. (F) Hepatogenic differentiation by ICG uptake and mRNA of albumin and TAT by qRT-PCR. Scale bar $=50 \mu \mathrm{m}$. Data from each group are expressed as the mean \pm SD. ${ }^{*} p<0.05$ versus undifferentiated (Undiff). 
A

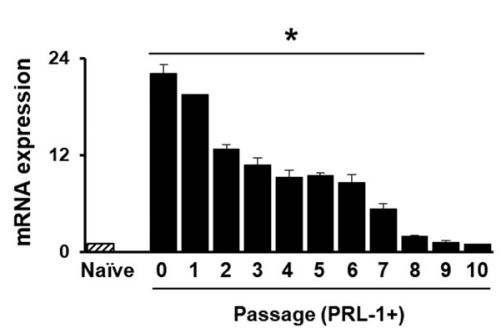

C

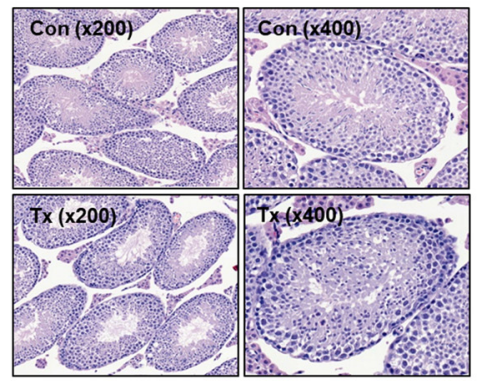

B

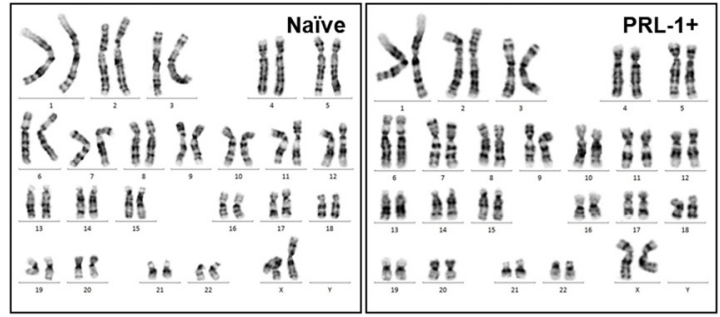

D

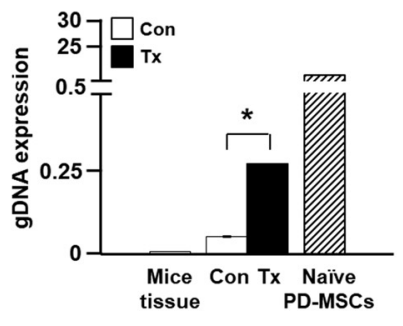

Fig. 3. Safety evaluation of PD$\mathrm{MSCS}^{\mathrm{PRL}-1}$ using the nonviral electroporation technique. (A) mRNA expression of PRL-1 in PD-MSCs ${ }^{\text {PRL-1 }}$ from 10 passages by qRT-PCR. * $p$ $<0.05$ versus naïve. (B) Karyotyping of naïve and PD-MSCs ${ }^{\text {PRL-1 }}$. (B) H\&E staining in normal (Con) and PDMSCPRL-1 transplanted testes (Tx) in NOD/SCID mice. (C) Human Alu expression in gDNA from each testis. Data from each group are expressed as the mean \pm SD. ${ }^{*} \mathrm{p}<0.05$ versus Con.

\section{Safety evaluation of PD-MSCs ${ }^{\text {PRL-1 }}$ using the nonviral electroporation technique}

The expression of PRL-1 in PD-MSCs ${ }^{\text {PRL-1 }}$ was significantly increased until passage 8 compared to that of the naive PD-MSCs (Fig. 3A, ${ }^{*} \mathrm{p}<0.05$ ). The karyotypes of the naive and PD-MSCs ${ }^{\text {PRL-1 }}$ were diploid and stable (Fig. 3B). In NOD/SCID mouse testes, there was no teratoma formation (Fig. 3C), and human Alu expression was significantly increased after PD-MSC ${ }^{\text {PRL-1 }}$ transplantation (Fig. 3D, $* p<0.05$ ). Therefore, these data suggest that PD-MSCs ${ }^{\text {PRL-1 }}$ generated by a nonviral electroporation system showed efficacy as well as safety.

\section{Conclusion}

An optimized protocol for PD-MSCs ${ }^{\text {PRL-1 }}$ using nonviral AMAXA electroporation was established, and its efficiency was confirmed. In addition, the characteristics of PD-MSCs ${ }^{\text {PRL-1 }}$ were similar to those of the naive MSCs, and a safety evaluation was accomplished. Therefore, this nonviral electroporation technique could be a new strategy for the clinical application of stem cell therapy.

\section{Acknowledgments}

This research was supported by a grant of the Korea Health Technology R\&D Project through the Korea Health Industry Development Institute (KHIDI), funded by the Ministry of Health \& Welfare, Republic of Korea (grant number: HI16C1559) and by a grant (18172MFDS1 82) from Ministry of Food and Drug Safety in 2020.

\section{Potential Conflict of Interest}

The authors have no conflicting financial interest.

\section{Supplementary Materials}

Supplementary data including one figure can be found with this article online at https://doi.org/10.15283/ijsc20117

\section{References}

1. Park JS, Suryaprakash S, Lao YH, Leong KW. Engineering mesenchymal stem cells for regenerative medicine and drug delivery. Methods 2015;84:3-16

2. Ricks DM, Kutner R, Zhang XY, Welsh DA, Reiser J. Optimized lentiviral transduction of mouse bone marrowderived mesenchymal stem cells. Stem Cells Dev 2008;17: 441-450

3. Ye Z, Lu W, Liang L, Tang $M$, Wang Y, Li Z, Zeng H, Wang A, Lin M, Huang L, Wang H, Hu H. Mesenchymal stem cells overexpressing hepatocyte nuclear factor-4 alpha alleviate liver injury by modulating anti-inflammatory functions in mice. Stem Cell Res Ther 2019;10:149

4. Oggu GS, Sasikumar S, Reddy N, Ella KKR, Rao CM, Bokara KK. Gene delivery approaches for mesenchymal stem cell therapy: strategies to increase efficiency and specificity. Stem Cell Rev Rep 2017;13:725-740

5. Santos JL, Pandita D, Rodrigues J, Pêgo AP, Granja PL, Tomás H. Non-viral gene delivery to mesenchymal stem cells: methods, strategies and application in bone tissue engineering and regeneration. Curr Gene Ther 2011;11:46-57

6. Lee HJ, Choi JH, Jung J, Kim JK, Lee SS, Kim GJ. Changes in PTTG1 by human TERT gene expression modulate the self-renewal of placenta-derived mesenchymal 
stem cells. Cell Tissue Res 2014;357:145-157

7. Kim MJ, Shin KS, Jeon JH, Lee DR, Shim SH, Kim JK, Cha DH, Yoon TK, Kim GJ. Human chorionic-plate-derived mesenchymal stem cells and Wharton's jelly-derived mesenchymal stem cells: a comparative analysis of their potential as placenta-derived stem cells. Cell Tissue Res 2011; 346:53-64

8. Lee MJ, Jung J, Na KH, Moon JS, Lee HJ, Kim JH, Kim GI, Kwon SW, Hwang SG, Kim GJ. Anti-fibrotic effect of chorionic plate-derived mesenchymal stem cells isolated from human placenta in a rat model of $\mathrm{CCl}(4)$-injured liver: potential application to the treatment of hepatic diseases. J Cell Biochem 2010;111:1453-1463

9. Lee HJ, Cha KE, Hwang SG, Kim JK, Kim GJ. In vitro screening system for hepatotoxicity: comparison of bonemarrow-derived mesenchymal stem cells and Placenta-derived stem cells. J Cell Biochem 2011;112:49-58` 